Economía TeOría y PrÁctica • Nueva Época, número 47, julio-diciembre 2017,

pp. 65-98, http://dx.doi.org/10.24275/ETYPUAM/NE/472017/Hernandez

\title{
Capacidades tecnológicas y organizacionales de las empresas mexicanas participantes en la cadena de valor de la industria aeronáutica*
}

\section{Technological and organizational capabilities of the Mexican companies participating in the aircraft industry value chain}

\author{
Juana Hernández Chavarria**
}

\begin{abstract}
RESUMEN
El clima actual del mercado global ha generado nuevas competencias y retos para las empresas en todo el mundo. El sector aeronáutico es un sector dinámico en innovaciones, I+D y uso de nuevos materiales, que hacen más eficiente el producto final. Este trabajo describe las capacidades tecnológicas y organizacionales de las empresas mexicanas que tienen participación en la cadena de valor; dichas capacidades son centrales en el posicionamiento actual y futuro de las empresas, ya que es primordial continuar aprendiendo para cubrir los requerimientos específicos que demanda el sector. Los resultados muestran que las empresas que poseen mejores capacidades son de tamaño mediano, tienen una capacidad productiva diversificada, clientes en distintos países del mundo y la mayoría proviene del sector de maquinados. Se encontró que las capacidades organizacionales son fundamentales en el proceso de inserción a la industria aeronáutica y determinan las actividades iniciales dentro del sector, en mayor medida que las tecnológicas.
\end{abstract}

Palabras clave: Capacidades tecnológicas, capacidades organizacionales, pymes, industria aeronáutica, México.

Clasificación JEL: L62

\begin{abstract}
The current climate of global market has generated new skills and challenges for companies worldwide. The aviation industry is a dynamic sector in innovation, $R \& D$ and use of new materials, which make more efficient the final product. This paper describes the technological and organizational capabilities of Mexican companies in the value chain; these capabilities are central to the current and future positioning of companies, it is essential to continue learning to meet the specific requirements demanded by the sector. The results show that companies that have better capabilities are medium size, have a diversified production capacity, customers in countries around the world and most comes from machined industry. It was found that organizational skills are essential in the insertion process to the aircraft industry and determine the initial activities within the sector, greater extent than technological.
\end{abstract}

Keywords: Technological Capabilities, Organizational Capabilities, sMEs, Aircraft Industry, México. JEL Classification: L62

* Fecha de recepción: 02/11/2016. Fecha de aprobación: 02/03/2017. Este trabajo se realizó en el marco del programa de becas posdoctorales de la UNAM.

** Universidad Nacional Autónoma de México, México. Correo electrónico: juana. hernandez@flacso.edu.mx. ORCID 0000-0003-0268-6280 


\section{INTRODUCCIÓN}

El clima actual del mercado global ha generado nuevas competencias y retos para las empresas en todo el mundo. Las nuevas tecnologías para la organización flexible de la producción tienen gran importancia en la competitividad; el surgimiento de nuevos paradigmas organizativos ha creado transformaciones en la forma de producción y por ende en las actividades innovativas dentro de las empresas. El sector aeronáutico es un sector dinámico en innovaciones, investigación y desarrollo (I+D) y uso de nuevos materiales y procesos con apoyo de modernas tecnologías de la información y la comunicación que hacen más eficiente el producto final. Estas transformaciones ganan importancia en aspectos como: las estrategias de diferenciación de producto y proceso, las mejoras incrementales y radicales en productos y servicios, el cuidado del medio ambiente y el uso de nuevos materiales integrados en el paradigma de manufactura avanzada.

El rápido ritmo de la tecnología, la competencia y la globalización de los mercados crean un entorno donde sólo las empresas que sean capaces de aprender a un ritmo acelerado y continuo lograrán mantenerse con éxito (Ngwenya-Scoburgh, 2009). Las crecientes transformaciones en la organización de la producción impactan en el comportamiento de la demanda, en la forma en cómo se desarrollan los procesos de innovación, así como en el uso efectivo de las tecnologías y materiales en el desarrollo de innovaciones. En un sector como el aeronáutico, las empresas de recién ingreso necesitan trabajar en el desarrollo de capacidades que les permitan utilizar el conocimiento existente en el mercado y hacer un uso efectivo de la tecnología para competir con calidad en esta cadena de producción global.

Este trabajo describe las capacidades tecnológicas y organizacionales de las empresas mexicanas que sirven de proveedoras de empresas suministradoras de aeropartes y/o fabricantes de aviones dentro de la cadena de valor global de la industria aeronáutica, dichas capacidades son centrales en el posicionamiento actual y futuro de las empresas, es primordial continuar aprendiendo para cubrir los requerimientos específicos que demanda el sector. Aunque el nivel de inserción de empresas mexicanas proveedoras aún es bajo, se han identificado Pymes locales que están realizando actividades productivas con éxito en esta industria, han logrado responder a las demandas productivas, organizacionales y requerimientos particulares de la industria.

Se trata de un trabajo pionero, dada la juventud de la industria en México. Analiza 15 empresas mexicanas ubicadas en los estados de Baja California, Nuevo León, Querétaro, Jalisco, Estado de México, Chihuahua y San Luis Potosí. Las interrogantes que guían el trabajo son: 1) ¿Qué nivel de capacidades tecnológicas y organizacionales tienen las empresas mexicanas participantes en la cadena de 
valor de la industria aeronáutica y cuáles son sus características?, 2) ¿Cómo las capacidades contribuyen a mejorar y estimular la posición competitiva, el nivel de inserción y las posibilidades de escalamiento (upgrade) en la cadena de proveeduría de esta industria?

\section{REVISIÓN DE ESTUDIOS PREVIOS SOBRE LA INDUSTRIA AERONÁUTICA EN MÉXICO}

El sector aeronáutico está considerado dentro del paradigma de manufactura avanzada, se encarga del diseño, desarrollo, fabricación, ensamble, comercialización, reparación y venta de aeronaves. El ciclo del producto consiste en el desarrollo de $\mathrm{I}+\mathrm{D}$, el diseño, la fabricación, el ensamble, ensayos y certificación, y por último las actividades de mantenimiento (López et al., 2012). El mercado aeronáutico se divide en tres sectores, según sus funciones y ámbitos de operación: civil, militar y comercial.

La industria aeronáutica ${ }^{1}$ se caracteriza por grandes inversiones y ciclos de producción muy largos donde el alto nivel tecnológico en el diseño y manufactura, la complejidad del sistema productivo y la multiplicidad de disciplinas que tienen injerencia en la producción, determinan una gestión descentralizada de la producción dominada por las grandes armadoras que operan a nivel internacional (Casalet, 2013). Es además una industria que se caracteriza por una estructura piramidal dominada por empresas líderes manufactureras de equipo original (Original Equipment Manufacturer, oEM) (Niosi y Zhegu, 2005).

La presencia de la industria aeronáutica en México tiene un siglo, aunque alcanzó su auge y prosperidad en la última década con la llegada de inversiones de empresas transnacionales a distintas regiones del país. Sin embargo, la industria no alcanzó un despuente significativo sino hasta principios del siglo XXI. Actualmente se tiene un crecimiento anual del 16 por ciento en promedio (Ruiz, 2016). A raíz del crecimiento reciente, diversos autores han realizado estudios en distintos temas sobre el sector en México, principalmente en el centro y el norte del país. En el norte, Carrillo y Hualde $(2013,2007)$ resaltan la importancia que juega el capital humano dentro de esta industria y plantean la importancia de la capacitación, la vinculación y el desarrollo de instituciones educativas que ayuden a satisfacer las demandas de la industria y formar recursos humanos competitivos. Contreras (2012), y Contreras y Bracamonte (2013) han analizado la paulatina acumulación de capacidades tecnológicas de las empresas sonorenses en ramas como la electrónica, metalmecánica y automotriz, que han sido importantes para la atracción de

\footnotetext{
${ }^{1}$ Para efectos de este trabajo se mencionará, indistintamente, la industria aeronáutica y aeroespacial como sinónimos.
} 
empresas extranjeras de la industria aeroespacial, una rama industrial emergente dentro de la entidad, que ha atraído la atención de diversos grupos académicos y de la administración pública ante los beneficios potenciales que representa para la región (Urbina, 2010: 2).

Contreras y Bracamonte (2013) adicionalmente analizan las capacidades de manufactura global en Sonora. Dicho estudio encontró, entre otras cosas, que las empresas establecidas se dedican en su mayoría a procesos de maquinados, subensambles y fabricación de componentes menores, es decir, operaciones de tercer y cuarto nivel. El crecimiento del sector en Sonora ha sido impulsado por las estrategias de descentralización productiva de las transnacionales y por una activa política de atracción de inversiones por parte del gobierno del estado.

En el centro del país, principalmente en el estado de Querétaro, se han realizado diversos estudios para comprender la complejidad de este sector y su evolución. Se han analizado las capacidades y oportunidades para el desarrollo de la industria aeronáutica y cómo la llegada de una empresa transnacional, en este caso Bombardier (empresa canadiense), a mediados de la década pasada, ha detonado cambios en la dinámica industrial del estado (Villavicencio et al., 2013; Hernández, 2010).

Casalet (2011) analiza los diferentes actores y redes públicas y privadas en el desarrollo del sector aeroespacial en el clúster de Querétaro. Se plantea que en la formación de este agrupamiento sectorial y regional se han plasmado redes de carácter multifuncional, cuyos propósitos se relacionan con varios factores, con variabilidad técnica e introducción de nuevos métodos de manufactura y comercial. Dichas redes funcionan como una estructura de negociación, posibilitando a los participantes el diseño de nuevos productos, muestra los numerosos actores y redes que trabajan para impulsar el desarrollo de esta industria a nivel nacional e internacional, en temas como: certificaciones, formación de recursos humanos calificados, marco institucional, desarrollo tecnológico, productividad, y sobre todo en ayudar a las empresas mexicanas a insertarse en el sector como proveedores directos.

A nivel nacional se analizan y describen diversas iniciativas que se han gestado en México, tanto del gobierno local como nacional, para fomentar el desarrollo de capacidades productivas y tecnológicas locales que impacten en la posición del país a nivel mundial. La creación de instituciones educativas como la Universidad Nacional Aeronáutica de Querétaro (UNAQ) y acuerdos como el Acuerdo Bilateral de Seguridad Aérea (BASA), y la Alianza para la Seguridad y la Prosperidad de América del Norte, significaron responder a las demandas a nivel de la certificación para la producción de empresas locales (Casalet, 2011; Femia, 2012).

Estudios de carácter internacional sugieren que México es un potencial ganador en industrias como la aeronáutica, por el crecimiento que ha mostrado en los últimos años y por su papel en el suministro de América del Norte; debido a las 
ventajas de frontera con Estados Unidos, y las condiciones que brinda el Tratado de Libre Comercio de América del Norte (TLCAN) (BCG, 2011). Sin embargo, reflexiones de estudios a nivel nacional, como el de Brown y Domínguez (2013), y Hernández (2015), muestran evidencia de la débil inserción de México en la cadena global de valor, se observa que el país tiene participación, pero sólo mediante las empresas transnacionales instaladas en el país. Se adolece de una estrategia exitosa para la inserción de empresas locales en dicha cadena, y existen muy pocas empresas mexicanas que realizan actividades en esta industria. Se plantea que se ha tenido un desgajamiento de cadenas productivas con encadenamientos poco sólidos, ya que muchas de las capacidades logradas durante la sustitución de importaciones, a partir de la apertura comercial, se han perdido o están muy debilitadas, lo que pone al país en una situación de desventaja para responder a las demandas tan particulares como las de la aeronáutica. Además de los estudios anteriores, otros estudiosos se han interesado en analizar el significado en este sector; se muestra que la construcción social del significado del trabajo ha pasado por un proceso de transformación, debido principalmente a la fragmentación productiva de la cadena de valor a escala internacional (Soto, 2014).

En cuanto a capital humano, Hernández (2016) muestra la creciente necesidad de capital humano altamente calificado para afrontar los cambios en la forma de fabricación, en base a procesos digitales y multidisciplinarios en el marco de un trabajo de colaboración durante todo el ciclo de vida del producto.

Los estudios que se han descrito de manera breve, permiten tener una perspectiva del desarrollo del sector aeronáutica en México; además sirven como elemento de justificación para este trabajo, ya que no se han realizado estudios a nivel micro sobre las características de las empresas mexicanas proveedoras del sector, ni sobre las capacidades tecnológicas y organizacionales que han desarrollado, por lo cual este texto resulta pertinente.

\section{CONCEPTUALIZACIÓN DE LAS CAPACIDADES TECNOLÓGICAS Y ORGANIZACIONALES}

Para este trabajo se entenderá por capacidades organizacionales a las actividades y habilidades que tienen las empresas para organizar, coordinar, controlar y lograr las tareas, en términos productivos y administrativos, que demanda la empresa de manera eficaz (Chandler, 1990; Velásquez y Ceballos, 2008; Anderson, 2009; Zollo y Winter, 2002; Boonpattarakan, 2012; Stacey, 2003; Degravel, 2011).

Dichas capacidades ayudan a la empresa a resolver de manera satisfactoria problemas que surgen en el proceso de introducción y uso de la tecnología, producción y servicios, búsqueda de recursos financieros, humanos y de información para el 
logro de la calidad en productos, servicios y procesos que permitan mantener y lograr la estrategia corporativa (Velásquez y Ceballos, 2008; Boonpattarakan, 2012).

Las capacidades organizacionales dan cuenta de las posibilidades de una real apropiación social de las tecnologías, y de su posterior mejoramiento y transformación. Desde la perspectiva de los recursos y capacidades, se entiende que detrás de la innovación como proceso empresarial están las capacidades organizacionales que la hacen posible (Velásquez y Ceballos, 2008; Boonpattarakan, 2012).

En cuanto a las capacidades tecnológicas, son la forma en que las empresas identifican, adoptan, usan, dominan, modifican y/o crean tecnologías y hacen uso de conocimiento nuevo o existente para la elaboración de nuevos productos y mejora en productos y procesos (OECD, 2006; Dutrénit, 2000; Lall, 1992; Cohen y Levinthal, 1990; Villavicencio, 1994; Lundvall y Johnson, 1994; Utepi, 2007; Villavicencio et al., 1995).

Las empresas mediante el uso, generación y absorción de conocimiento y aprendizaje logran un impacto en el manejo efectivo de la tecnología, desempeño organizacional, mejoras en la capacidad de gestión y métodos de producción. Para el logro de las capacidades tecnológicas las empresas necesitan actividades de inversión y producción en equipos, infraestructura, bienes de capital, recursos humanos calificados y conocimiento codificado mediante patentes, manuales, entre otros (Schumpeter, 1989; Katz, 2015; Torres, 2006).

\section{II.1 Capacidades tecnológicas}

El rápido ritmo de la tecnología, la competencia y la globalización de los mercados crean un entorno donde sólo las empresas que sean capaces de aprender a un ritmo acelerado y continuo, lograrán mantenerse con éxito (Ngwenya-Scoburgh, 2009). Las crecientes transformaciones en la organización de la producción impactan en el comportamiento de la demanda, en cómo se desarrollan los procesos de innovación, así como en el uso efectivo de las tecnologías y los materiales en el desarrollo de innovaciones.

Hoy en día existe un consenso sobre la importancia de la innovación en la creación de ventajas competitivas en las empresas, por ser un factor poderoso que explica las diferencias en el rendimiento entre éstas y en regiones y países; las empresas que tienen éxito en la innovación prosperan a expensas de sus competidores menos capaces y tienen mayor productividad e ingresos que los menos innovadores (Fagerberg, 2003).

El desarrollo de ciertas capacidades y acceso a recursos es fundamental para tener una posición competitiva, tanto de empresas grandes como pequeñas y medianas; asimismo, la capacidad de una nación para fomentar y gestionar el cambio tecnoló- 
gico es crucial para la creación de capacidades dentro de las empresas y para sobrevivir y crecer en el mercado internacional. El desarrollo de capacidades tecnológicas es el resultado de las inversiones realizadas por la empresa en respuesta a los estímulos externos e internos, y en la interacción con otros agentes económicos, tanto públicos como privados, nacionales y extranjeros (Lall, 1992).

Organismos internacionales como la Organización para la Cooperación y el Desarrollo Económicos (OECD) definen las capacidades tecnológicas como "los aprendizajes acumulados por las empresas, que les permiten mantener una dinámica innovadora y que teóricamente están estrechamente relacionadas con el desempeño organizacional" (OECD, 2006:15). La generación y acumulación de capacidades tecnológicas va más allá de lo que hagan las empresas individualmente, demanda tiempo y recursos e involucra fenómenos sistémicos de aprendizaje y de estrategias dinámicas de los gobiernos y la estructuración de un Sistema Nacional de Innovación (SNI), donde deben estar involucradas, además de las empresas, las universidades, las escuela técnicas, programas y políticas públicas selectivas y demás instituciones que tengan influencia en la creación y difusión de conocimientos tecnológicos en la sociedad (OECD, 2006).

Las capacidades tecnológicas son procesos de aprendizaje acumulados; el aprendizaje es la forma de adquirir nuevo conocimiento que ayude al manejo de la tecnología, mejora en la capacidad de gestión y los métodos de producción y organización.

El aprendizaje es fundamental para la construcción de capacidades tecnológicas, se entiende como cualquier forma de adquisición o adaptación de conocimiento. Aplicado a una empresa se refiere a la forma en que crea o aumenta su capacidad de gestión y manejo de la tecnología. Por lo tanto el aprendizaje es la habilidad para acumular conocimiento, que al mismo tiempo ayuda al uso y empleo de mayor aprendizaje, necesario para la creación y el fortalecimiento de capacidades tecnológicas (OECD, 2006).

Los procesos de aprendizaje permiten a las organizaciones acumular y desplegar capacidades tecnológicas centrales; el aprendizaje es la forma en que se obtiene conocimiento nuevo, se acumula conocimiento previo y se crean condiciones para retroalimentar el proceso. El aprendizaje dado en forma interna, se genera en las actividades desarrolladas por la organización, como es el caso de la producción, I+D, el marketing, entre otros (Dutrénit, 2000).

Cohen y Levinthal (2007), en su estudio sobre la innovación y el aprendizaje, como las dos implicaciones de la doble función de la $\mathrm{I}+\mathrm{D}$, resaltan su importancia para generar innovaciones y desarrollar la capacidad de la empresa para identificar, asimilar y explotar el conocimiento disponible en el medio ambiente; es decir, para generar aprendizaje o capacidades de absorción. 
Estos autores distinguen tres fuentes de conocimiento tecnológico utilizado por una empresa: $i$ ) las actividades de I+D propias de la empresa; ii) el conocimiento originado por las derramas de actividades de I+D de los competidores, iii) el conocimiento que se origina fuera de la industria. La facilidad del aprendizaje o la capacidad de absorción da cuenta de la influencia del gasto en $\mathrm{I}+\mathrm{D}$, de la apropiabilidad de conocimientos y delinea las condiciones de oportunidad tecnológica y ventaja competitiva de la empresa (Cohen y Levinthal, 2007).

Las capacidades de absorción son una función del nivel de educación y de la permeabilidad de los empleados, la infraestructura tecnológica y el apoyo a la gestión; son esenciales para desarrollar y mantener las capacidades tecnológicas productivas y organizacionales (Cohen y Levinthal, 1990).

La emergencia de las capacidades tecnológicas y del aprendizaje como un proceso de acumulación de las mismas surge a principios de la década de $1980 \mathrm{y}$ a partir de ahí se han desarrollado diversos trabajos en torno a su definición y medición. Para Katz (2007), el desarrollo de capacidades tecnológicas locales tiene que ver con el diseño de nuevos productos, el mejoramiento de tecnologías de procesos, el desarrollo de nuevas rutinas de organización de la producción y la captación de proveedores.

Las empresas construyen y acumulan capacidades tecnológicas a través de procesos de aprendizaje. En las empresas de países en desarrollo se plantea que estos procesos se adquieren de la tecnología que se obtiene de empresas de otros países, principalmente de países desarrollados (Torres, 2006).

Las empresas aprenden a lo largo del tiempo, acumulando conocimiento tecnológico, sobre esa base pueden emprender progresivamente nuevas actividades, y de esta forma adquirir nuevas capacidades. El aprendizaje organizacional se produce cuando el conocimiento se comparte, adapta, desarrolla, e introduce en los procedimientos de la organización, la estructura y la cultura; el aprendizaje continuo ayuda al mejoramiento del uso de habilidades generales y las del personal (Dodgson, 1993).

En una propuesta taxonómica Bell y Pavitt (1993) clasifican las principales capacidades tecnológicas por medio de cuatro funciones técnicas: dos básicas y dos de apoyo. Las básicas se refieren a las actividades de inversión y producción. Las funciones de apoyo se refieren al desarrollo de vínculos con empresas e instituciones y a la producción de bienes de capital. Mediante esta taxonomía se observan los niveles de capacidades tecnológicas que se definen por el grado de dificultad de las actividades; van desde los niveles más básicos de las capacidades de producción rutinarias, hasta niveles de profundidad como las capacidades de innovación. 
Bell y Pavitt (1993) también proponen cinco categorías para clasificar a las empresas de acuerdo con sus posibilidades de cambio técnico y a la construcción de capacidades tecnológicas:

i) Empresas dominadas por el proveedor. Donde el cambio técnico proviene de los proveedores de máquinas y otros insumos de producción. En esta categoría se encuentran sectores como el textil y el agrícola; la acumulación tecnológica y el aprendizaje tecnológico surgen de mejorar y manipular los métodos de producción.

ii) Empresas intensivas en escala. La acumulación de conocimiento y el aprendizaje tecnológico provienen de las mejoras en procesos y productos y se generan por el diseño, la operación y creación de complejos sistemas de producción y productos; las fuentes de mejoras tecnológicas son: el diseño, la ingeniería de producción, la experiencia operativa y los proveedores de equipos y componentes.

iii) Empresas intensivas en información. En este tipo de empresas la acumulación tecnológica se origina de la capacidad de diseñar, construir, operar y mejorar sistemas de almacenamiento y procesamiento de la información.

iv) Empresas basadas en la ciencia. En este caso la acumulación de tecnología y el proceso de aprendizaje se origina en las actividades de Investigación y Desarrollo (I+D) y finalmente,

v) Empresas de proveedores especializados. La acumulación tecnológica se origina del diseño, construcción y uso de los insumos de producción como las maquinarías, componentes, instrumentos y software (Bell y Pavitt, 1995).

Por su parte, Chesbrough (2006) desde la postura de la innovación abierta (Open Innovation) plantea que las empresas innovadoras extraen conocimiento de una gran variedad de fuentes y vínculos externos, y los integran a sus propias rutinas y procesos de aprendizaje, logrando con ello capacidades tecnológicas más avanzadas. El concepto de Sistema Nacional de Innovación (SNI) busca exponer el papel de dichos vínculos externos para explicar las dinámicas de desarrollo; la definición del SNI hace hincapié en las interacciones institucionales, vistas como una red de los sectores público y privado, cuyas actividades e interacciones inician, importan, modifican y difunden nuevas tecnologías (Freeman, 1987).

Asimismo, tienen influencia el tamaño de la empresa, el acceso a las competencias del mercado, la capacidad de organización y de gestión en la empresa y su capacidad de cambio en las estructuras para absorber nuevos métodos y tecnologías (Katz, 2007, 1987). 
A continuación, se presenta un panorama de las capacidades organizacionales, las cuales pueden verse como complemento de las capacidades tecnológicas.

\section{II.2 CAPACIDADES ORGANIZACIONALES}

Las capacidades organizacionales (CO) se consideran un ingrediente vital para la competitividad de una empresa, son un recurso intangible y un elemento de éxito, así como fuente de ventaja competitiva. En las co es donde las empresas desarrollan sus fortalezas para aumentar la competitividad, contribuir al crecimiento y mejorar el desempeño organizacional (Boonpattarakan, 2012).

Las capacidades organizacionales (CO) pueden ser descritas como las habilidades de marketing, las habilidades de distribución, las capacidades de desarrollo de productos, la capacidad de organización, y así sucesivamente. Son capacidades generales potencialmente aplicables a las diferentes industrias. Para Chandler (1990) las capacidades organizacionales de una empresa son las instalaciones colectivas y las habilidades de los empleados, sobre todo la capacidad de la mediana y alta gerencia.

Estas capacidades influyen en la creación de redes de conocimiento, combinando personas y bienes que, en su conjunto, permitirán a las empresas llevar a cabo sus tareas asignadas de manera más eficaz (Boonpattarakan, 2012). Las capacidades de organización se pueden derivar de la comercialización, del know-how, de la flexibilidad, de la innovación continua, del aprendizaje organizacional, del procesamiento de información, de los sistemas de información, de los recursos humanos, de la calidad y de los sistemas internos (Boonpattarakan, 2012; Nonaka y Takeuchi, 1999).

Las capacidades organizacionales (CO) reflejan los puntos fuertes y la experiencia de las organizaciones para competir con éxito en el mercado, es una necesidad que todas las empresas, independientemente de su tamaño, tienen que desarrollar (Boonpattarakan, 2012).

Algunas fuentes de capacidades organizacionales son la construcción de mejores productos o servicios, el suministro de productos con precios competitivos y un enfoque centrado en la innovación o capacidad tecnológica para la mejora de la competitividad.

Las capacidades organizacionales (CO), por algunos autores llamadas "capacidades de gestión" o "capacidades operativas", se definen como la capacidad de una organización en términos de $a$ ) recursos humanos, tales como la calidad, las habilidades y competencias; $b$ ) recursos físicos y materiales como: máquinas, terrenos y edificios; $c$ ) recursos financieros como el dinero y el crédito, y e) recursos de información como el conocimiento y las bases de datos; así como las de 
innovación y recursos intelectuales, como derechos de autor, diseños y patentes (Boonpattarakan, 2012).

La capacidad de gestión ${ }^{2}$ contribuye a un mejor diseño e implementación de la estrategia empresarial, y facilita la comprensión de la industria y la generación de opciones estratégicas; hace referencia a la capacidad de la organización para administrar, coordinar, controlar y gobernar un conjunto de actividades (Stacey, 2003; Degravel, 2011).

Aunque la naturaleza de las capacidades organizacionales sigue siendo un tema debatido en la literatura especializada, es claro que están asociadas con las posibilidades de las empresas para abordar de manera satisfactoria la solución de los problemas que surgen en el proceso de introducción y utilización de una nueva tecnología. En este sentido, la existencia de capacidades organizacionales asegura la superación de los problemas que emergen en el proceso, lo que da como resultado la incorporación exitosa de la tecnología (Velásquez y Ceballos, 2008).

Bell (1984) hace una diferencia entre las capacidades organizacionales u operativas (aquellas que permiten mantener operativa una tecnología) de las capacidades tecnológicas (aquellas que permiten dominar y dirigir el cambio técnico). En consecuencia, es posible identificar distintos escenarios evolutivos del proceso de introducción de una nueva tecnología, según el nivel de capacidades que las organizaciones logren acumular en función de los distintos procesos de aprendizaje tecnológico (veáse Velásquez y Ceballos, 2008).

Tomer (1987) afirmó que la organización estratégica, la estrategia corporativa, la estructura organizativa, la cultura y los procedimientos de la organización son un reflejo de la capacidad organizativa. Aunque autores como Barney (1991) sugieren que la posesión de los recursos no es suficiente para crear ventajas competitivas, sino que las empresas tienen que organizarse y sacar el máximo provecho de ellos para alcanzar competitividad.

La construcción de capacidades organizacionales depende en gran medida de los recursos de la organización (los recursos financieros, de marketing, operativos y humanos), de la arquitectura organizativa (como el sistema de información sobre la planificación, el sistema de compensación, la cultura del aprendizaje y el trabajo en equipo) y de la calidad de la organización (calidad de los productos o servicios); las empresas que logren con eficacia y eficiencia desarrollar estos elementos tendrán capacidades organizacionales sólidas y podrán competir y tener éxito en el mercado a largo plazo (Boonpattarakan, 2012; Ulrich, 1990).

\footnotetext{
${ }^{2}$ Para efectos de este trabajo se tomará a las capacidades organizacionales y de gestión como sinónimos.
} 
Autores como Teece et al. (1997) clasifican a las capacidades organizacionales dentro de las capacidades dinámicas, que se definen como "la capacidad de una empresa para integrar, construir y reconfigurar competencias internas y externas para hacer frente a entornos en rápida evolución". Las capacidades dinámicas al igual que las tecnológicas y las productivas evolucionan a través de fenómenos de aprendizaje (Eisenhardt y Martin, 2000).

Por otro lado, Anderson (2009) explica que las capacidades organizacionales tienen sus raíces en el campo teórico del comportamiento organizacional, a través de la superposición de las relaciones entre las capacidades de absorción, las capacidades organizativas, el aprendizaje organizacional y el capital social dentro de la empresa.

Las capacidades no pueden ser fácilmente transferidas de una empresa a otra. Por lo tanto, las capacidades deben desarrollarse y adaptarse dentro de una organización, se desarrollan a través de las rutinas organizativas e implican el desarrollo, la recopilación y el intercambio de información; por lo tanto, el aprendizaje organizacional juega un papel importante en el desarrollo de capacidades organizacionales (Killen et al., 2008).

En este sentido, las rutinas se definen como patrones reconocibles, repetitivos de acción interdependientes realizadas por múltiples actores, reducen la incertidumbre, proporcionan estabilidad y economizan recursos cognitivos, son como un mecanismo de toma de decisiones cuando la empresa se enfrenta a eventos no rutinarios (Becker, 2004).

De esta manera, las capacidades de organización también son vistas como un patrón de actividad colectiva a través del cual la organización genera y modifica sus rutinas de funcionamiento en la búsqueda de la ventaja competitiva de forma sistemática (Killen et al., 2008). Las capacidades son el producto de todo el sistema de la organización, incluyendo la acumulación de habilidades, rutinas y procesos (Anderson, 2009).

Desde la perspectiva de los recursos y capacidades, se entiende que detrás de la innovación como proceso empresarial están las capacidades organizacionales que la hacen posible (Velásquez y Ceballos, 2008; Boonpattarakan, 2012).

Zollo y Winter (2002) plantean que las capacidades organizacionales son dinámicas y coevolucionan a través de tres tipos de mecanismos de aprendizaje: i) la acumulación de experiencia tácita; ii) el conocimiento explícito, y iii) la codificación del conocimiento explícito. La fortaleza de la inversión en el aprendizaje tácito y el desarrollo de mecanismos para desarrollar una capacidad dinámica efectiva varían en relación con el nivel de énfasis en la creación de las capacidades organizacionales dentro de cada empresa. 


\section{DEFINICIÓN DE LA MUESTRA, ESTRATEGIA DE INVESTIGACIÓN Y HERRAMIENTAS DE ANÁLISIS}

\section{III.1 Definición de la muestra}

Este estudio analiza empresas cien por ciento mexicanas que sirven de proveedoras de empresas suministradoras de aeropartes y/o fabricantes de aviones a nivel nacional e internacional. Estas empresas son proveedoras de materiales diversos para la industria aeronáutica. Provienen de sectores como maquinados de alta precisión, automotriz, eléctrico, electrónico, textil y automotriz.

En México se tienen registradas 300 empresas del sector aeronáutico, entre extranjeras y mexicanas. Del total de ellas se identificaron 30 de capital mexicano, de acuerdo con información de la Federación Mexicana de la Industria Aeronáutica (Femia), la Secretaría de Economía (SE), y de organismos locales en los estados; este dato representa el 10 por ciento de las empresas totales ubicadas en el país. Sin embargo, por cuestiones ajenas a la investigación (políticas de manejo de la información de las empresas principalmente) no se pudo obtener el cuestionario electrónico contestado de 30 de ellas, ni entrevistas y visitas, sólo se tuvo acceso a 15 , que representan el 50 por ciento de las locales identificadas.

Las empresas se encuentran distribuidas en distintos estados del territorio mexicano, por lo que se considera un estudio de carácter nacional más que regional, a pesar de que la mayoría de las empresas se ubican en dos regiones del país, el centro y el norte. Los estados donde se encuentran las empresas mexicanas analizadas son: Baja California, Chihuahua, Jalisco, Estado de México, Nuevo León, Querétaro y San Luis Potosí.

Tabla 1. Número de empresas mexicanas identificadas por estado, año 2014

\begin{tabular}{|l|c|}
\hline \multicolumn{1}{|c|}{ ESTADO } & NÚMERO DE EMPRESAS \\
\hline Nuevo León & 14 \\
\hline Querétaro & 7 \\
\hline Baja California & 6 \\
\hline Jalisco & 3 \\
\hline Estado de México & 2 \\
\hline Chihuahua & 1 \\
\hline San Luis Potosí & 1 \\
\hline Total & 34 \\
\hline
\end{tabular}

Fuente: Elaboración propia con datos de ProMéxico 2013, Secretaría de Economía 2014, Femia, 2013, citado en tesis doctoral "Empresas mexicanas en la cadena de valor de la IA-DCS-IX Generación, México, Flacso, 2015" (Hernández, 2015a). 


\section{III.2 La estrategia de investigación}

La estrategia de investigación que siguió este trabajo fue un estudio de caso exploratorio-explicativo con análisis cualitativo, las herramientas de investigación para la recolección de la información empírica fueron: 1) un cuestionario electrónico aplicado a las empresas objeto de estudio; 2) entrevistas a profundidad a empleados o empresarios y agentes de instituciones públicas y privadas vinculadas con la industria, y 3) información de fuentes secundarias para obtener datos que complementen a las anteriores.

\section{III.3 Herramientas para el análisis de los datos}

Las herramientas para el análisis de los datos obtenidos del cuestionario electrónico y las entrevistas fueron, por un lado, el programa estadístico informático SPSS ${ }^{3}$ y, por el otro, el método de la diferencia propuesto por Mill (1973). El programa SPSS es ampliamente utilizado en el estudio de las ciencias sociales, permite analizar información de bases de datos y brinda elementos para hacer inferencias. En cuanto al método de la diferencia, éste consiste en seleccionar casos positivos y negativos del objeto de estudio, lo que permite observar variación en la variable dependiente. En este caso los casos positivos son las empresas que posean capacidades sólidas, definidas por los indicadores propuestos en la tabla 2. Y los casos negativos son las empresas con capacidades débiles. Al seleccionar los casos positivos y negativos, se pueden conocer las características de las empresas con mejores capacidades y observar su nivel de inserción y con ello determinar si las capacidades son determinantes en su posición actual y posibilidades de escalamiento, pasa lo mismo con los casos negativos (o de capacidades débiles).

La lógica de inferencia de este procedimiento es que todos los casos que presentan el resultado de interés, en este caso que posean capacidades tecnológicas y/o organizacionales, deben también compartir una característica explicativa común, es decir, ser empresas mexicanas proveedoras de la aeronáutica, mientras que los casos negativos deben carecer del atributo de interés, es decir, no poseer capacidades. En este caso, no es que no tengan capacidades, sino que son débiles, ya que para insertarse a esta industria se debe contar con ciertos requerimientos que forzosamente demandan algún tipo de capacidades. Sin embargo, para este caso, puede que posean capacidades débiles, pero que aun así sean proveedoras del sector, lo que permite tener un ámbito mayor de explicación sobre los factores determinantes para ser proveedores de la aeronáutica.

\footnotetext{
${ }^{3}$ Statistical Package for the Social Sciences.
} 
El método de la diferencia servirá para conocer si las empresas poseen o no capacidades tecnológicas y organizacionales; para posteriormente encontrar las que, de acuerdo con los indicadores definidos, poseen capacidades sólidas o débiles, esto no necesariamente significa que no tenga el atributo (capacidades), sino que permite analizar a las empresas con mejores capacidades y a partir de ahí conocer sus características y nivel de inserción en la industria aeronáutica.

La siguiente tabla muestra las variables, indicadores y valores para la recolección de información que permitió identificar el nivel de capacidades tecnológicas y organizacionales de las empresas mexicanas proveedoras de industria aeronáutica.

Para obtener el dato sobre el nivel de capacidades tecnológicas y organizacionales por empresa se utilizó la información obtenida en las respuestas del cuestionario electrónico. De acuerdo con el tipo de pregunta se definió la forma de medición, como se muestra en la tabla 2, en la columna de valores. Para el caso de las preguntas cerradas dicotómicas ${ }^{4}$ el valor era uno para "sí" y cero para "no". Si la pregunta era de escala, se tomó en cuenta el cociente entre las opciones elegidas sobre las opciones posibles; y por último, si era de porcentaje, se definió una regla de tres, donde el porcentaje máximo era igual a uno.

Con estos criterios se generó un número para cada pregunta, donde a mayor valor obtenido son mejores las capacidades tecnológicas u organizacionales de la empresa y viceversa.

Tabla 2. Variables e indicadores para la recolección de información

\begin{tabular}{|c|c|c|}
\hline \multicolumn{3}{|c|}{ NIVEL MICRO } \\
\hline VARIABLE & INDICADORES & VALORES \\
\hline \multirow{4}{*}{$\begin{array}{c}\text { CAPACIDADES } \\
\text { TECNOLÓGICAS (cT) }\end{array}$} & Gastos en I+D & Sí = $1 \mathrm{No}=0$ \\
\hline & Departamento de I+D & $\mathrm{Sí}=1 \mathrm{No}=0$ \\
\hline & Innovaciones y mejoras en productos y procesos & Sí $=1 \mathrm{No}=0$ \\
\hline & $\begin{array}{l}\text { Tipo de actividades: } \\
\text { 1) Ingeniería } \\
\text { 2) Diseño y actividades de I+D } \\
\text { 3) Distribución } \\
\text { 4) Manufactura } \\
\text { 5) Soporte técnico } \\
\text { 6) Servicio al cliente } \\
\text { 7) Ensamble } \\
\text { 8) Tratamiento térmico } \\
\text { 9) Pintado /recubrimiento } \\
\text { 10) Otra }\end{array}$ & $\begin{array}{l}11 \text { = } 1 \text { (depende el número } \\
\text { de actividades se tiene el va- } \\
\text { lor igual o menor a } 1 \text { ) }\end{array}$ \\
\hline
\end{tabular}

${ }^{4}$ Las preguntas dicotómicas cerradas son las que tienen como posible respuesta un "Sí" o "No". 
80 ECONOMÍA TeORÍA Y PRÁCTICA • Nueva Época, número 47, julio-diciembre 2017

Tabla 2 (continuación)

\begin{tabular}{|c|c|c|}
\hline \multirow[t]{5}{*}{ VARIABLE } & INDICADORES & VALORES \\
\hline & $\begin{array}{l}\text { Mecanismos de transferencia de tecnología: } \\
\text { 1) Patentes } \\
\text { 2) Marcas } \\
\text { 3) Secreto industrial } \\
\text { 4) Licencia de procesos } \\
\text { 5) Licencia de productos } \\
\text { 6) Otra }\end{array}$ & $\begin{array}{l}6=1 \text { (depende el número de } \\
\text { actividades se tiene el valor } \\
\text { igual o menor a } 1 \text { ) }\end{array}$ \\
\hline & $\begin{array}{l}\text { Inversión física } \\
\text { 1) Compra de maquinaria } \\
\text { 2) Compra de maquinaria y equipo } \\
\text { 3) Adaptaciones en las instalaciones } \\
\text { 4) Otra }\end{array}$ & $\begin{array}{l}4=1 \text { (depende el número de } \\
\text { actividades se tiene el valor } \\
\text { igual o menor a } 1 \text { ) }\end{array}$ \\
\hline & $\begin{array}{l}\text { Escolaridad de los recursos humanos } \\
\text { 1) Primaria completa } \\
\text { 2) Secundaria completa } \\
\text { 3) Técnico } \\
\text { 4) Licenciatura } \\
\text { 5) Ingeniero } \\
\text { 6) Posgrado }\end{array}$ & $\begin{array}{l}6=1 \text { (depende el número de } \\
\text { actividades se tiene el valor } \\
\text { igual o menor a } 1 \text { ) }\end{array}$ \\
\hline & Idiomas del personal (principalmente inglés) & Sí $=1 \mathrm{No}=0$ \\
\hline \multirow[t]{4}{*}{$\begin{array}{c}\text { CAPACIDADES } \\
\text { ORGANIZACIONALES } \\
\text { (CO) }\end{array}$} & $\begin{array}{l}\text { Programas de mejora de la calidad: } \\
\text { 1) Prácticas de mejora continua } \\
\text { 2) Equipos de trabajo } \\
\text { 3) Six sigma } \\
\text { 4) Programas de certificación de competencias la- } \\
\text { borales } \\
\text { 5) Otro }\end{array}$ & $\begin{array}{l}5=1 \text { (depende el número de } \\
\text { actividades se tiene el valor } \\
\text { igual o menor a 1) }\end{array}$ \\
\hline & Programas de modernización organizacional & Sí = $1 \mathrm{No}=0$ \\
\hline & $\begin{array}{l}\text { Capacidad para gestionar fondos y créditos } \\
\text { 1) Fondos gubernamentales federales } \\
\text { 2) Fondos gubernamentales estatales } \\
\text { 3) Préstamos bancarios } \\
\text { 4) Préstamos privados } \\
\text { 5) Otro }\end{array}$ & $\begin{array}{l}5=1 \text { (depende el número de } \\
\text { actividades se tiene el valor } \\
\text { igual o menor a } 1 \text { ) }\end{array}$ \\
\hline & $\begin{array}{l}\text { Actividades de adaptación y mejora } \\
\text { 1) Compra de maquinaria } \\
\text { 2) Compra de maquinaria y equipo } \\
\text { 3) Adaptaciones en las instalaciones } \\
\text { 4) Otra }\end{array}$ & $\begin{array}{l}4=1 \text { (depende el número de } \\
\text { actividades se tiene el valor } \\
\text { igual o menor a } 1 \text { ) }\end{array}$ \\
\hline
\end{tabular}

Fuente: Elaboración propia, tomada de la tesis doctoral "Las empresas mexicanas en la cadena de valor de la industria aeronáutica, Flacso, 2015" ( Hernández, 2015a). 


\section{DESCRIPCIÓN DE LAS EMPRESAS MEXICANAS PARTICIPANTES EN LA CADENA DE VALOR DE LA INDUSTRIA AERONÁUTICA}

En el año 2003 se empezó formalmente una política de atracción de inversiones de la industria aeronáutica a México. Tanto el gobierno federal como estatal (destacan los estados de Querétaro, Baja California, Estado de México y Chihuahua) realizaron acciones para atraer inversores. Dichos estados contaban con capacidades y vocaciones productivas que ofrecían experiencia y capital humano calificado para esta industria. Es una industria relativamente nueva en el país, pero que ha tenido un crecimiento sostenido a lo largo de poco más de una década.

La industria aeronáutica en México está en constante crecimiento, actualmente existen alrededor de 300 empresas de la industria aeronáutica (entre extranjeras y mexicanas), y se encuentra ubicada en el lugar 15 como proveedor de la industria a nivel mundial (AeroStrategy, 2009).

La creciente actividad del sector aeronáutico en México puede tener una explicación en las ventajas que ofrece en cuanto a localización geográfica, por su cercanía con el mercado más importante (Estados Unidos), lo que implica una reducción de costos de producción principalmente de las compañías que realizan operaciones en Europa. La experiencia y nivel de competitividad alcanzados en otros sectores como el automotriz y el electrónico permite contar con una base de personal y empresas que pueden orientarse al sector aeronáutico y tratados de libre comercio que permiten el acceso en condiciones preferenciales en 43 mercados (Secretaría de Economía, 2012).

Las empresas mexicanas estudiadas son proveedoras de materiales diversos, su experiencia previa es principalmente en el área de maquinados, aunque también resaltan sectores como el textil, el eléctrico-electrónico y el automotriz. Los productos que proveen a la industria tienen orientaciones diferentes, en actividades de propulsión, maquinados de precisión, fabricación de componentes para turbinas, partes de electrónica y aeroestructuras (sheet metal), forjas, componentes para sistema de aterrizaje, partes plásticas y tratamiento superficiales. Adicionalmente, se producen partes de material compuesto, ingeniería y diseño de interiores de avión (asientos), cabinas y cocinetas.

Las empresas que se analizaron por estado y su tamaño ${ }^{5}$ se muestran en la tabla 3. Los estados donde se abarcó el total de empresas mexicanas son

${ }^{5}$ Para definir el tamaño de las empresas como micro, pequeñas y medianas (Pymes), se tomó como base la estratificación o clasificación propuesta por la Secretaría de Hacienda y Crédito Público (SHCP) (SHCP, 2014). La SHCP hace la clasificación de acuerdo al sector, en este caso se tomó el sector de industria y servicios. Donde, de acuerdo al rango de trabajadores, se determina el tamaño 
Chihuahua y San Luis Potosí. Dichos estados resultan interesantes; el caso de Chihuahua por presentar dinamismo, crecimiento y presencia en este sector, pero con baja inserción de empresas locales. Para el caso de San Luis Potosí, se tienen identificadas pocas empresas en general y es interesante que haya presencia local dentro de su territorio.

En Baja California y Jalisco se cubrió el 67 por ciento del total, para el caso de Querétaro se tuvo acceso al 57 por ciento de las empresas mexicanas localizadas. En Nuevo León se alcanzó el 36 por ciento, aunque es el porcentaje más bajo, al ser el estado con mayor número de empresas mexicanas en el sector, este porcentaje es representativo y equivale a las empresas cubiertas en Baja California y Querétaro.

Tabla 3. Porcentaje total de empresas mexicanas estudiadas por estado

\begin{tabular}{|c|c|c|}
\hline ESTADO & TAMAÑO DE LA EMPRESA & \% DE EMPRESAS CUBIERTAS \\
\hline \multirow{3}{*}{ Baja California } & Grande & 16.7 \\
\hline & Mediana & 16.7 \\
\hline & Pequeña & 33.5 \\
\hline \multicolumn{2}{|l|}{ Total } & 67 \\
\hline \multirow{3}{*}{ Nuevo León } & Grande & 18 \\
\hline & Mediana & 9 \\
\hline & Pequeña & 9 \\
\hline \multicolumn{2}{|l|}{ Total } & 36 \\
\hline Chihuahua & Mediana & 100 \\
\hline \multicolumn{2}{|l|}{ Total } & 100 \\
\hline \multirow{3}{*}{ Querétaro } & Mediana & 14.2 \\
\hline & Pequeña & 28.5 \\
\hline & Micro & 14.2 \\
\hline \multicolumn{2}{|l|}{ Total } & 57 \\
\hline San Luis Potosí & Pequeña & 100 \\
\hline \multicolumn{2}{|l|}{ Total } & 100 \\
\hline Jalisco & Pequeña & 67 \\
\hline \multicolumn{2}{|l|}{ Total } & 67 \\
\hline
\end{tabular}

Fuente: Elaboración propia, citada en la tesis doctoral "Las empresas mexicanas en la cadena de valor de la industria aeronáutica, Flacso, 2015" (Hernández, 2015a).

La tabla anterior muestra que el 46.7 por ciento de las empresas bajo estudio son pequeñas, es decir, tienen entre 11 y 50 empleados; el 26.7 por ciento son me-

de la empresa. Hasta 10 trabajadores es micro, desde 11 hasta 50 es pequeña y de 51 hasta 250 trabajadores es mediana. Más de 250 trabajadores es considerada grande. 
dianas con entre 51 y 250 empleados, y el 20 por ciento son grandes con más de 250 empleados.

El caso de las microempresas, con menos de 10 empleados, son las de porcentaje menor con el 6.7 por ciento, lo que hace pensar que a mayor tamaño de las empresas, más oportunidades de integrarse a este sector, lo cual podría ser comprensible debido a los requerimientos y la complejidad de éste. Sin embargo, en este caso la mayoría de las empresas son pequeñas, lo que sugiere que existen otros factores, además del tamaño, que influyen en la inserción.

Tabla 4. Tamaño de las empresas mexicanas estudiadas de la aeronáutica

\begin{tabular}{|l|c|c|}
\hline TAMAÑO & $\%$ & \% ACUMULADO \\
\hline Micro & 6.7 & 6.7 \\
Pequeña & 46.7 & 53.3 \\
Mediana & 26.7 & 80.0 \\
Grande & 20.0 & 100.0 \\
Total & 100.0 & \\
\hline
\end{tabular}

Fuente: Elaboración propia, citada en la tesis doctoral "Las empresas mexicanas en la cadena de valor de la industria aeronáutica, Flacso, 2015" (Hernández, 2015a).

\section{CAPACIDAdES TECNOLÓGiCAS Y ORGANIZACIONALES DE LAS EMPRESAS MEXICANAS PARTICIPANTES EN LA CADENA DE VALOR DE LA INDUSTRIA AERONÁUTICA}

\section{V.1 Las capacidades tecnológicas}

Las empresas mediante el uso, generación, absorción de conocimiento y aprendizaje logran un impacto en el manejo efectivo de la tecnología, desempeño organizacional, mejoras en la capacidad de gestión y métodos de producción. Las capacidades tecnológicas permiten la generación y circulación de nuevo conocimiento, que está estrechamente ligado con las características del capital humano que poseen las empresas y la forma en que se organiza el trabajo productivo.

En el sector aeronáutico la organización de la producción es un proceso complejo, cuya intensidad depende de: $i$ ) la necesidad de resolver problemas concretos en situación de incertidumbre; ii) demanda de soluciones rápidas que en ocasiones no pueden ser codificables; iii) la complejidad de la tecnología, y iv) en algunos procesos los saberes técnicos están dados y demandan un proceso de aprendizaje mayor para las empresas de nuevo ingreso.

La medición de las capacidades tecnológicas de las empresas mexicanas de la aeronáutica se hizo en función de los indicadores que se enumeraron en la tabla 2. 
En la gráfica 1 se muestra el resultado para todas las empresas; con el método de la diferencia se identificó a las empresas mexicanas con mejores capacidades. En general las capacidades tecnológicas de las empresas mexicanas son bajas, este comportamiento quizá se deba a que las actividades que realizan no demandan manipulación de tecnología de punta. La organización y complejidad del sector hace que la intensidad tecnológica y la innovación se desarrollen en eslabones de la cadena dominados por las empresas líderes en el sector a nivel mundial.

La gráfica 1 permite identificar a las empresas con mejores capacidades y las de menor nivel, aunque la diferencia entre ellas es muy alto: también permite conocer sus características y tener elementos explicativos de su influencia en el nivel de inserción y oportunidades de desarrollo para las empresas locales. El valor más alto para definir capacidades tecnológicas sólidas está dado por el número 6, este valor se obtuvo de los valores definidos en la tabla 1.

En la tabla 5 se rescatan los casos positivos y negativos, (de acuerdo a Mill, 1973), es decir las empresas con capacidades sólidas y las de capacidades débiles.

Las empresas con mejores capacidades tecnológicas dentro del sector aeronáutico son empresas de tamaño mediano, pequeño y micro, no aparece ninguna empresa grande. Es una empresa mediana la mejor posicionada. Proviene del sector textil y del vestido, realiza gastos en $\mathrm{I}+\mathrm{D}$ que se han materializado en desarrollos propios, cuenta con una patente, un secreto industrial en proceso y licencias de productos y procesos.

\section{Gráfica 1. Capacidades tecnológicas de las empresas mexicanas de la aeronáutica}

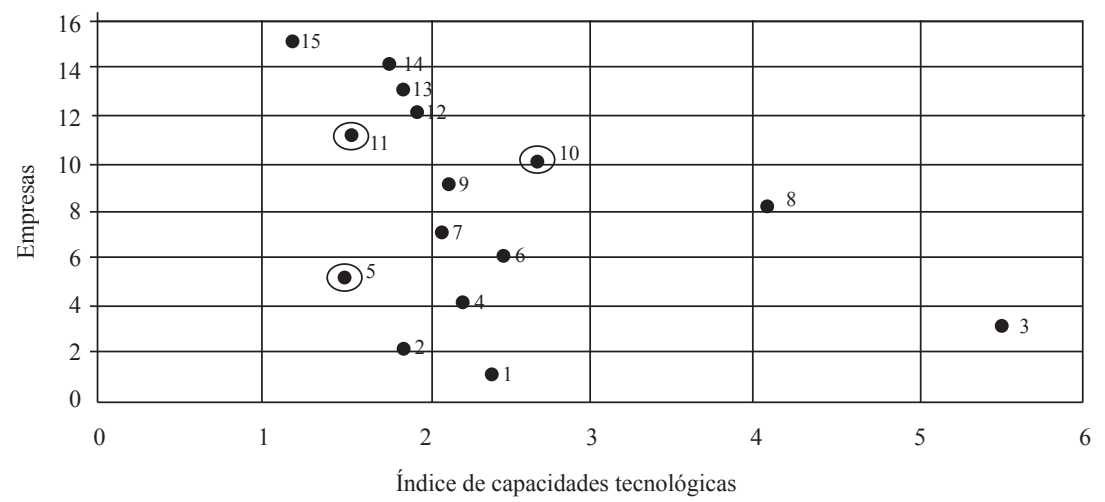

Fuente: Elaboración propia con base en método de la diferencia, citada en la tesis doctoral "Las empresas mexicanas en la cadena de valor de la industria aeronáutica, Flacso, 2015" (Hernández, 2015a). 
Tabla 5. Capacidades tecnológicas de las empresas mexicanas de la aeronáutica

\begin{tabular}{|c|c|c|}
\hline EMPRESA & CAPACIDADES SÓLIDAS & CAPACIDADES DÉBILES \\
\hline 3 & + & - \\
\hline 5 & + & \\
\hline 8 & + & \\
\hline 10 & & - \\
\hline 11 & & - \\
\hline 15 & & \\
\hline
\end{tabular}

Fuente: Elaboración propia, citada en la tesis doctoral "Las empresas mexicanas en la cadena de valor de la industria aeronáutica, Flacso, 2015" ( Hernández, 2015a).

A pesar de que el promedio de escolaridad es de nivel primaria, esta empresa ha logrado consolidar capacidades tecnológicas. Las inversiones en I+D le han permitido identificar y dominar ciertas tecnologías que la han llevado a tener desarrollos propios y un buen nivel de inserción dentro de la aeronáutica.

En cuanto a las empresas pequeñas y medianas que muestran capacidades tecnológicas sólidas, ambas provienen del sector de maquinados. Son empresas que invierten en $\mathrm{I}+\mathrm{D}$, aunque no han logrado desarrollos propios, realizan prácticas de mejora continua que complementan el esfuerzo en I+D. La estrategia de estas empresas para mantenerse en el sector está enfocada a recursos humanos, diferenciación y costos y flexibilidad.

Tabla 6. Características de las empresas mexicanas de la aeronáutica con capacidades tecnológicas sólidas

\begin{tabular}{|c|c|c|c|c|}
\hline EMPRESA & TAMAÑO & EXPERIENCIA PREVIA & ESTRATEGIA PARA ENTRAR AL SECTOR & $\begin{array}{l}\text { ESTRATEGIA PARA MANTENER- } \\
\text { SE DENTRO DELL SECTOR }\end{array}$ \\
\hline 3 & MEDIANA & $\begin{array}{l}\text {-Sector textil } \\
\text {-Inició operaciones a } \\
\text { partir del } 2000 \\
\text {-Promedio de escolari- } \\
\text { dad: primaria } \\
\text {-Implementa prácticas } \\
\text { de mejora continua }\end{array}$ & \begin{tabular}{|l|}
-Redes profesionales/programas \\
gubernamentales \\
Datos actuales en el sector \\
-Invierte en I+D \\
- Cuenta con mecanismos de trans- \\
ferencia tecnológica como: paten- \\
te, secreto industrial en proceso, \\
marca, licencia de procesos y de \\
productos \\
-Actualmente tiene desarrollos pro- \\
pios dentro del sector \\
-No cuenta con ningún ingeniero \\
dentro de su platilla de producción \\
\end{tabular} & RH/diferenciación \\
\hline
\end{tabular}


Tabla 6 (continuación)

\begin{tabular}{|c|c|c|c|c|}
\hline EMPRESA & TAMAÑO & EXPERIENCIA PREVIA & ESTRATEGIA PARA ENTRAR AL SECTOR & $\begin{array}{c}\text { ESTRATEGIA PARA MANTENER- } \\
\text { SE DENTRO DEL SECTOR }\end{array}$ \\
\hline 8 & MICRO & $\begin{array}{l}\text {-Sector maquinados y } \\
\text { otros } \\
\text {-Inició operaciones a } \\
\text { partir del } 2000 \\
\text {-Promedio de escolari- } \\
\text { dad: licenciatura } \\
\text {-Implementa prácticas } \\
\text { de mejora continua }\end{array}$ & $\begin{array}{l}\text {-Diversificación } \\
\text { Datos actuales en el sector } \\
\text {-Inversión en I+D } \\
\text {-No cuenta con mecanismos de } \\
\text { transferencia tecnológica } \\
\text {-No cuenta con desarrollos propios } \\
\text {-Cuenta con aproximadamente } 10 \\
\text { ingenieros }\end{array}$ & Costos y flexibilidad \\
\hline 10 & PEQUEÑA & $\begin{array}{l}\text {-Sector maquinados y } \\
\text { otros } \\
\text {-Inició operaciones a } \\
\text { partir del } 2000 \\
\text {-Promedio de escolari- } \\
\text { dad: técnicos con es- } \\
\text { tudios superiores } \\
\text {-Implementa prácticas } \\
\text { de mejora continua }\end{array}$ & $\begin{array}{l}\text { Redes profesionales/programas gu- } \\
\text { bernamentales } \\
\text { Datos actuales en el sector } \\
\text {-No tiene inversión en I+D } \\
\text {-No cuenta con mecanismos de } \\
\text { transferencia tecnológica } \\
\text {-No tiene desarrollo propios } \\
\text {-Cuenta con aproximadamente } 10 \\
\text { ingenieros }\end{array}$ & RH/diferenciación \\
\hline
\end{tabular}

Fuente: Elaboración propia, tomada de la tesis doctoral "Las empresas mexicanas en la cadena de valor de la industria aeronáutica, Flacso, 2015" (Hernández, 2015a).

Las empresas mexicanas con capacidades tecnológicas débiles también son empresas medianas y una pequeña, al igual que en las capacidades tecnológicas sólidas, lo que sugiere que estas capacidades no son determinantes en la inserción al sector aeronáutico. A diferencia de las productivas que se consideran determinantes en la posición de las empresas, ya que mientras las empresas logren cumplir con los requerimientos y la calidad que demanda el cliente podrán diversificar su producción.

Tabla 7. Características de las empresas mexicanas de la aeronáutica con capacidades tecnológicas débiles

\begin{tabular}{|c|c|c|c|c|}
\hline EMPRESA & TAMAÑO & EXPERIENCIA PREVIA & ESTRATEGIA PARA ENTRAR AL SECTOR & $\begin{array}{c}\text { ESTRATEGIA PARA } \\
\text { MANTENERSE }\end{array}$ \\
\hline 5 & MEDIANA & $\begin{array}{l}\text {-Sector eléctrico/electró- } \\
\text { nico } \\
\text {-Inició operaciones a } \\
\text { partir del } 2000 \\
\text {-Promedio escolaridad: } \\
\text { técnicos con estudios } \\
\text { superiores } \\
\text {-Implementa prácticas } \\
\text { de mejora continua }\end{array}$ & $\begin{array}{l}\text {-Empleado trabajó en una empresa de la } \\
\text { aeronáutica/el cliente lo contacto } \\
\text { Datos actuales en el sector } \\
\text {-No invierte en I+D } \\
\text {-No implementa mecanismos de transfe- } \\
\text { rencia tecnológica } \\
\text {-No cuenta con desarrollos propios } \\
\text {-Cuenta con aproximadamente } 10 \text { inge- } \\
\text { nieros }\end{array}$ & Costos y flexibilidad \\
\hline
\end{tabular}


Tabla 7 (continuación)

\begin{tabular}{|c|c|c|c|c|}
\hline EMPRESA & TAMAÑO & EXPERIENCIA PREVIA & ESTRATEGIA PARA ENTRAR AL SECTOR & $\begin{array}{l}\text { ESTRATEGIA PARA } \\
\text { MANTENERSE }\end{array}$ \\
\hline 11 & PEQUEÑA & $\begin{array}{l}\text {-Sector maquinados y } \\
\text { otros } \\
\text {-Inició operaciones a } \\
\text { partir del } 2000 \\
\text {-Promedio de escolari- } \\
\text { dad: licenciatura } \\
\text {-Implementa prácticas } \\
\text { de mejora continua }\end{array}$ & $\begin{array}{l}\text {-Empleado trabajó en una empresa de la } \\
\text { aeronáutica/el cliente lo contacto } \\
\text { Datos actuales en el sector } \\
\text {-No invierte en I+D } \\
\text {-No implementa mecanismos de transfe- } \\
\text { rencia tecnológica } \\
\text {-No cuenta con desarrollos propios } \\
\text {-Cuenta con aproximadamente } 10 \text { inge- } \\
\text { nieros }\end{array}$ & $\begin{array}{l}\text { Cercanía con el cliente/ } \\
\text { tiempos de entrega }\end{array}$ \\
\hline 15 & MEDIANA & $\begin{array}{l}\text {-Sector maquinados y } \\
\text { otros } \\
\text {-Inició operaciones a } \\
\text { partir del } 2000 \\
\text {-No implementa prácti- } \\
\text { cas de mejora continua }\end{array}$ & $\begin{array}{l}\text { Datos actuales en el sector } \\
\text {-No invierte en I+D } \\
\text {-No implementa mecanismos de transfe- } \\
\text { rencia tecnológica } \\
\text {-No cuenta con desarrollos propios } \\
\text {-Cuenta con aproximadamente } 10 \text { inge- } \\
\text { nieros }\end{array}$ & $\begin{array}{l}\text { Cercanía con el cliente/ } \\
\text { tiempos de entrega }\end{array}$ \\
\hline
\end{tabular}

Fuente: Elaboración propia, citada en la tesis doctoral "Las empresas mexicanas en la cadena de valor de la industria aeronáutica, Flacso, 2015" (Hernández, 2015a).

El análisis anterior sugiere que las actividades que realizan las empresas mexicanas (hasta ahora) no demandan capacidades tecnológicas fuertes, aunque es importante que las empresas fortalezcan dichas capacidades para, en un futuro cercano, lograr mejoras en procesos y productos que impacten en su posición actual dentro de la cadena de producción.

En el sector aeronáutico las empresas de recién ingreso necesitan trabajar en el desarrollo de capacidades que les permiten utilizar el conocimiento existente en el mercado y hacer un uso efectivo de la tecnología para poder competir con calidad en una cadena de producción global.

\section{V.2 Las capacidades organizacionales de las empresas mexicanas participantes en la cadena de valor de la aeronáutica}

Las capacidades organizacionales ayudan a la empresa a resolver de manera satisfactoria problemas que surgen en el proceso de introducción y uso de la tecnología, producción y servicios, búsqueda de recursos financieros, humanos y de información para el logro de la calidad en productos, servicios y procesos que permitan mantener y lograr la estrategia corporativa.

Para identificar las capacidades organizacionales que poseen las empresas mexicanas de la aeronáutica, se tomaron como base programas de mejora de la 
calidad que han implementado, los programas de modernización organizacional que les permitieron ser más eficientes en el manejo de recursos y respuesta al cliente, y la capacidad de gestión de fondos y créditos que provean a la empresa de recursos económicos para el logro de los objetivos productivos y las actividades de adaptación y mejora en cualquier área de la empresa.

La gráfica 2 muestra las capacidades organizacionales para cada una de las 15 empresas bajo estudio y la tabla 8 rescata las empresas con mejores capacidades, de las cuales se retoman las características más importantes.

Gráfica 2. Capacidades organizacionales de las empresas mexicanas de la aeronáutica

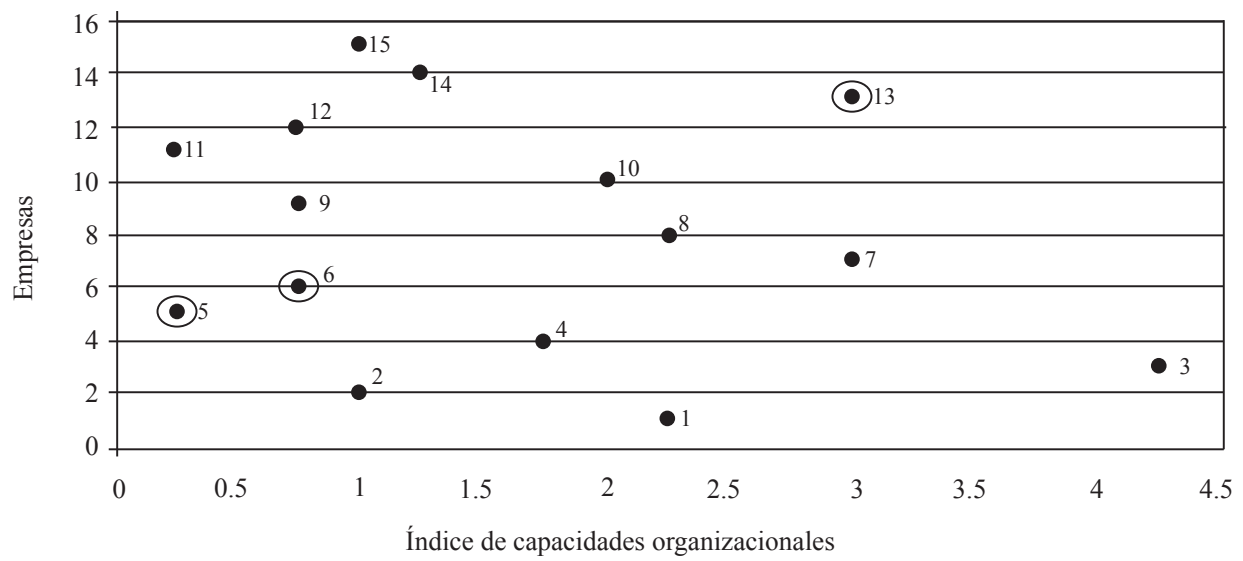

Fuente: Elaboración propia, citada en la tesis doctoral "Las empresas mexicanas en la cadena de valor de la industria aeronáutica, Flacso, 2015" (Hernández, 2015a).

Tabla 8. Empresas mexicanas de la aeronáutica con capacidades organizacionales sólidas y débiles

\begin{tabular}{|c|c|c|}
\hline EMPRESA & CAPACIDADES SOLLDAS & CAPACDADES DÉBILES \\
\hline 3 & + & \\
\hline 5 & & - \\
\hline 6 & + & - \\
\hline 7 & & - \\
\hline 11 & + & \\
\hline 13 & & \\
\hline
\end{tabular}

Fuente: Elaboración propia, citada en la tesis doctoral "Las empresas mexicanas en la cadena de valor de la industria aeronáutica, Flacso, 2015" (Hernández, 2015a). 
Las empresas con mejores capacidades organizacionales son medianas y pequeñas; las empresas medianas provienen del sector textil y de maquinados, son empresas que han implementado programas de modernización organizacional y mejora continua de la calidad de sus productos y procesos. Además tienen la capacidad de gestionar fondos y créditos sin recurrir a consultores externos, lo que contribuye a acceder a recursos económicos para cubrir las necesidades del sector y aumentar las posibilidades de escalamiento.

La empresa pequeña que se identificó proviene del sector automotriz y al igual que las medianas ha logrado gestionar recursos externos e implementar programas de mejora en actividades de la empresa.

Las capacidades organizacionales de estas empresas muestran que poseen habilidades para realizar actividades productivas con calidad, que son capaces de buscar mecanismos (recursos económicos) para tener una mejor posición e invertir en recursos humanos calificados, y mecanismos para satisfacer las necesidades de sus clientes.

Son empresas que siguen distintas estrategias para permanecer en el sector, que van desde recursos humanos calificados y diferenciación de sus productos; cercanía con el cliente, y tiempos de entrega hasta costos y flexibilidad.

Pasando a las empresas con capacidades organizacionales débiles, se observa que todas son empresas pequeñas, que no han logrado consolidar programas enfocados a la modernización organizacional y a la mejora continua de la calidad en sus productos y procesos. Una posible explicación es que son empresas que apenas están en proceso de certificación y no cuentan con las habilidades y recursos necesarios para consolidar otras áreas de la empresa y gestionar recursos externos.

En suma, las empresas medianas son las que mejores capacidades poseen, lo cual también pone en evidencia que son éstas las que mejor nivel de inserción tienen en la cadena de valor de la industria aeronáutica.

Retomando a Bell y Pavitt (1993), las empresas mexicanas de la aeronáutica son empresas dominadas por el proveedor, ya que la tecnología y las máquinas provienen de fuentes externas, además el desarrollo de nuevos procesos y productos son dados por el cliente, principalmente por las armadoras que dominan la industria a nivel internacional. Para el caso de estas empresas la acumulación tecnológica y el aprendizaje surgen de mejorar y manipular métodos de producción, lo que contribuye a consolidar las capacidades tecnológicas con amplio uso de las capacidades organizacionales para el dominio de la tecnología y el desarrollo de nuevos aprendizajes que les permitan escalar en la cadena productiva.

Los resultados muestran que las empresas que poseen mejores capacidades son de tamaño mediano, tienen una capacidad productiva diversificada, clientes 
Tabla 9. Características de las empresas mexicanas con capacidades organizacionales sólidas

\begin{tabular}{|c|c|c|c|c|}
\hline EMPRESA & TAMAÑO & $\begin{array}{c}\text { EXPERIENCIA } \\
\text { PREVIA }\end{array}$ & $\begin{array}{l}\text { ESTRATEGIA PARA } \\
\text { ENTRAR AL SECTOR }\end{array}$ & $\begin{array}{l}\text { ESTRATEGIA PARA } \\
\text { MANTENERSE }\end{array}$ \\
\hline 3 & MEDIANA & $\begin{array}{l}\text {-Sector textil } \\
\text {-Inició operaciones a partir } \\
\text { del } 2000 \\
\text {-Promedio de escolaridad: } \\
\text { técnicos con estudios su- } \\
\text { periores } \\
\text {-Implementa prácticas de } \\
\text { mejora continua }\end{array}$ & $\begin{array}{l}\text {-Redes profesionales/pro- } \\
\text { gramas gubernamentales. } \\
\text { Datos actuales en el sector } \\
\text {-Cuentan con programas } \\
\text { de modernización organi- } \\
\text { zacional } \\
\text {-Han gestionado fondos y } \\
\text { créditos con personal de la } \\
\text { empresa }\end{array}$ & RH/diferenciación \\
\hline 7 & PEQUEÑA & $\begin{array}{l}\text {-Sector automotriz. } \\
\text {-Inició operaciones a partir } \\
\text { del } 2000 \\
\text {-Promedio de escolaridad: } \\
\text { técnicos con estudios su- } \\
\text { periores } \\
\text {-Implementa prácticas de } \\
\text { mejora continua }\end{array}$ & $\begin{array}{l}\text {-Empleado trabajó en una } \\
\text { empresa de la aeronáutica/ } \\
\text { el cliente lo contacto } \\
\text { Datos actuales en el sector } \\
\text {-Cuentan con programas } \\
\text { de modernización organi- } \\
\text { zacional } \\
\text {-Han gestionado fondos y } \\
\text { créditos con personal de la } \\
\text { empresa }\end{array}$ & $\begin{array}{l}\text { Cercanía con el clien- } \\
\text { te/ tiempos de entrega }\end{array}$ \\
\hline 13 & MEDIANA & $\begin{array}{l}\text {-Sector maquinados y } \\
\text { otros } \\
\text {-Inició operaciones a partir } \\
\text { del } 2000 \\
\text {-Promedio de escolaridad: } \\
\text { técnicos con estudios su- } \\
\text { periores } \\
\text {-Implementa prácticas de } \\
\text { mejora continua }\end{array}$ & $\begin{array}{l}\text { Redes profesionales/pro- } \\
\text { gramas gubernamentales } \\
\text { Datos actuales en el sector } \\
\text {-Cuentan con programas } \\
\text { de modernización organi- } \\
\text { zacional } \\
\text {-Han gestionado fondos y } \\
\text { créditos con personal de la } \\
\text { empresa }\end{array}$ & Costos y flexibilidad \\
\hline
\end{tabular}

Fuente: Elaboración propia, citada en la tesis doctoral "Las empresas mexicanas en la cadena de valor de la industria aeronáutica, Flacso, 2015" (Hernández, 2015a).

en distintos países del mundo y la mayoría proviene del sector de maquinados. Se encontró que las capacidades organizacionales son fundamentales en el proceso de inserción y determinan las actividades iniciales dentro del sector, en mayor medida que las tecnológicas. Estas empresas provienen, en gran parte, de empresas exitosas en otros sectores en donde adquirieron habilidades específicas que son valiosas en la industria aeronáutica. Una de ellas es la industria de maquinados que posee mayor convergencia en experiencia y conocimientos para la 
Tabla 10. Características de las empresas mexicanas de la aeronáutica con capacidades organizacionales débiles

\begin{tabular}{|c|c|c|c|c|}
\hline EMPRESA & TAMAÑO & EXPERIENCIA PREVIA & ESTRATEGIA PARA ENTRAR AL SECTOR & $\begin{array}{c}\text { ESTRATEGIA PARA } \\
\text { MANTENERSE }\end{array}$ \\
\hline 5 & PEQUEÑA & $\begin{array}{l}\text {-Sector eléctrico/electrónico } \\
\text {-Inició operaciones a partir del } \\
2000 \\
\text {-Promedio escolaridad: técnicos } \\
\text { con estudios superiores } \\
\text {-Implementa prácticas de mejo- } \\
\text { ra continua }\end{array}$ & $\begin{array}{l}\text {-Empleado trabajó en una } \\
\text { empresa de la aeronáutica/el } \\
\text { cliente lo contacto } \\
\text { Datos actuales en el sector } \\
\text {-No cuentan con programas } \\
\text { de modernización organiza- } \\
\text { cional } \\
\text {-No han gestionado fondos } \\
\text { y/o créditos }\end{array}$ & \begin{tabular}{|l|} 
Costos y \\
flexibilidad
\end{tabular} \\
\hline 6 & PEQUEÑA & $\begin{array}{l}\text {-Sector eléctrico/electrónico } \\
\text {-Inició operaciones a partir del } \\
2000 \\
\text {-Promedio escolaridad: técnicos } \\
\text { con estudios superiores } \\
\text {-Implementa prácticas de mejo- } \\
\text { ra continua }\end{array}$ & $\begin{array}{l}\text {-Empleado trabajó en una } \\
\text { empresa de la aeronáutica/el } \\
\text { cliente lo contacto } \\
\text { Datos actuales en el sector } \\
\text {-No cuentan con programas } \\
\text { de modernización organiza- } \\
\text { cional } \\
\text {-No han gestionado fondos } \\
\text { y/o créditos }\end{array}$ & $\begin{array}{l}\text { Cercanía con el } \\
\text { cliente/ tiempos } \\
\text { de entrega }\end{array}$ \\
\hline 11 & PEQUEÑA & $\begin{array}{l}\text {-Sector maquinados y otros } \\
\text {-Inició operaciones a partir del } \\
2000 \\
\text {-Promedio de escolaridad: licen- } \\
\text { ciatura } \\
\text {-Implementa prácticas de mejo- } \\
\text { ra continua }\end{array}$ & $\begin{array}{l}\text {-Empleado trabajó en una } \\
\text { empresa de la aeronáutica/el } \\
\text { cliente lo contacto } \\
\text { Datos actuales en el sector } \\
\text {-No cuentan con programas } \\
\text { de modernización organiza- } \\
\text { cional } \\
\text {-No han gestionado fondos } \\
\text { y/o créditos }\end{array}$ & $\begin{array}{l}\text { Cercanía con el } \\
\text { cliente/ tiempos } \\
\text { de entrega }\end{array}$ \\
\hline
\end{tabular}

Fuente: Elaboración propia, citada en la tesis doctoral "Las empresas mexicanas en la cadena de valor de la industria aeronáutica, Flacso, 2015" (Hernández, 2015a).

aeronáutica, aunque también se identifican algunas que provienen del sector textil y del vestido y la automotriz.

Adicionalmente, otro hallazgo, derivado de las entrevistas realizadas en el trabajo de campo, fue que, además de alianzas estratégicas, las empresas medianas son las que más vinculación tienen con agentes externos estratégicos como: laboratorios privados, universidades, centros públicos de investigación, secretarías de desarrollo económico a nivel estatal, y la SE y ProMéxico a nivel federal. 


\section{Conclusiones}

La juventud de la industria aeronáutica mexicana obliga a las empresas a invertir en la acumulación de capacidades para lograr colocarse como proveedoras de la industria aeronáutica que les permita escalar en la cadena. Los resultados muestran que hay gran heterogeneidad entre las empresas y que son las de tamaño mediano las que poseen mejores capacidades tecnológicas y organizacionales, en comparación con las pequeñas y grandes. Esto no quiere decir que no haya empresas grandes con capacidades, pero no son la mayoría.

Estas empresas producen diversidad de productos para el sector y han logrado tener variedad de clientes en distintos países del mundo. Provienen, en gran parte, de empresas exitosas en otros sectores en donde adquirieron habilidades específicas que son valiosas en la industria aeronáutica. Uno de ellos es la industria de maquinados que posee mayor convergencia en experiencia y conocimientos para el aeronáutico, aunque también hay algunas que provienen del sector textil y del vestido y de la industria automotriz.

Las empresas medianas implementan programas de modernización organizacional, lo que les ha permitido desarrollar capacidades sólidas para gestionar fondos gubernamentales y créditos privados, factor importante para mejorar su posición dentro del sector y acceder a nuevas certificaciones para diversificar producción.

En cuanto a las estrategias para mantenerse en el sector, priorizan los recursos humanos calificados con experiencia en otros sectores, los costos, la flexibilidad, la cercanía con el cliente y los tiempos de entrega. Mantienen estímulos económicos para el personal de manera permanente y han logrado tener alianzas estratégicas con empresas transnacionales.

Otro hallazgo del trabajo de campo fue que además de las alianzas estratégicas, las empresas medianas son las que más vinculación tienen con agentes externos estratégicos como: laboratorios privados, universidades, centros públicos de investigación, secretarías de desarrollo económico a nivel estatal, y la SE y ProMéxico a nivel federal.

En cuanto a la segunda interrogante, este estudio muestra que las capacidades productivas y organizacionales son fundamentales en el proceso de inserción y son las que han sido determinantes para la participación de las empresas en la cadena de proveeduría en eslabones mejor posicionados, en la medida que les otorga flexibilidad para tener capacidad de respuesta a las demandas de productos y la calidad necesaria. Esto les permite acceder a mayores oportunidades para seguir escalando hacia otras actividades gracias a la experiencia que se va generando.

Ahora bien, una vez que las empresas están dentro de la cadena productiva del sector, son las capacidades tecnológicas las que contribuyen a mejorar su nivel de 
inserción y posibilidades de escalamiento a nivel horizontal y vertical; de acuerdo a los hallazgos de este estudio, las capacidades tecnológicas no juegan un papel central, ya que, como se ha evidenciado, las actividades productivas que realizan las empresas mexicanas no demandan uso intensivo de tecnología, sino más bien lo que importa son las habilidades del personal y la experiencia previa para absorber el conocimiento y cumplir con los requerimientos del cliente.

Este trabajo, sin duda tiene algunas limitaciones, en particular el tamaño de la muestra que representa la mitad de las empresas mexicanas presentes en la industria, ya que es frecuente que en un trabajo de campo de este tipo, las empresas prefieran no dar información. Sin embargo, la importancia de las empresas entrevistadas permite afirmar que el estudio da luces sobre el estado de las empresas de capital mexicano. Este es un estudio pionero y, por supuesto, es necesario profundizar en la investigación e incorporar nuevas líneas de investigación como podría ser un análisis a profundidad del desarrollo de la cadena de valor de la industria aeronáutica en México.

\section{REFERENCIAS BIBLIOGRÁFICAS}

AeroStrategy (2009), Implications for Canada's Aerospace Industry A Discussion Paper, Canadá.

Anderson, Kimberly (2009), Organizational capabilities as predictors of effective knowledge management: an empirical examination by Submitted to $H$. Wayne Huizenga, School of Business and Entrepreneurship Nova Southeastern University.

Barney, Jay (1991), "Firm resources and sustained competitive advantage", Journal of Management, 17, pp. 99-120.

Becker, Marcus (2004), "Organizational routines: a review of the literature", Industrial and Corporate Change, 13 (núm. 4), pp. 643-676.

Bell, Markus (1984), "And the Accumulation of Industrial Technological Capacity in Developing Countries", Technological Capacity in the Third World, Londres, Macmillan, pp. 187-209.

Bell, Martín y Keith, Pavitt (1993), "Technological Accumulation and Industrial Growth: contrast between developed and developing countries", Industrial and Corporate Change, vol. 2, núm. 2.

Boonpattarakan, Apicha (2012) "Model of Thai Small and Medium Sized Enterprises' Organizational Capabilities: Review and Verification", Journal of Management Research, 4 (3), pp. 15-43. http://doi.org/10.5296/jmr. v4i3.1557 
Boston Consuilting Group (BCG) (2011), Made in America, Again. Why Manufacturing Will Return to the U.S.

Brown Flor y Domínguez, Lilia (2013), "¿Tiene la industria aeronáutica mexicana las condiciones para integrarse a la cadena de valor internacional de alto valor agregado?", en Flacso-México (ed.), La industria aeroespacial: complejidad productiva e institucional, México, pp. 135-162.

Carrillo Jorge y Alfredo Hualde (2013), ¿Una maquila diferente? Competencias laborales profesionales en la industria aeroespacial en Baja California", en Flacso-México (ed.), La industria aeroespacial: complejidad productiva e institucional, México, pp. 163-198.

Casalet, Mónica (2011), Evolución y complejidad en el desarrollo de encadenamientos productivos en México: Los desafios de la construcción del cluster aeroespacial en Querétaro, México.

(2013), La industria aeroespacial, complejidad productiva e institucional, Flacso (ed.), México.

Chandler, Alred DuPont (1990), Scale and Scope:The Dynamics of Industrial Capitalism. Cambridge, (B. P. Harvard University Press, Ed.).

Chesbrough, Henry (2006), Open Innovation: A New Paradigm for Understanding Industrial Innovation.

Cohen, Wesley y Levinthal, Daniel (1990), "Absorptive capacity", Administrative Science Quarterly, 35, pp. 128-153. (2007), "Innovation and Learning: the Two Faces of RyD", The Economic Journal, 99 (397), pp. 569-596.

Contreras, Oscar y Bracamonte, Álvaro (2013), "Capacidades de manufactura global en regiones emergentes. La industria aeroesoacial en Sonora", en Flacso-México (ed.), La industria aeroespacial: complejidad productiva e institucional, México, pp. 199-223.

Contreras, Oscar (2012), "El ocaso de las maquiladoras y los desafíos de la política industrial en México", en C. N. de Universitarios (ed.), Nueva estrategia de industrialización, México, p. 335.

Degravel, Daniel (2011), "Managing organizational capabilities: the Keystone step", Journal of Strategy and Management, 4 (3), pp. 251-274. http://doi. org/10.1108/17554251111152270

Dodgson, Mark (1993), "Organizational Learning- A Review of some literaturesDodgson.pdf", Science Policy Research Unit, University of Sussex, Brighton, U.K., 14 (3), pp. 375-394. 
Dutrénit, Gabriela (2000), Learning and Knowledge Management in the Firm: From Knowledge Accumulation to Strategic Capabilities, Cheltenham (ed.), México.

Eisenhardt, Kathleen y Jeffrey, Martin (2000), "Dynamic capabilities: what are they?", Strategic Management Journal, 21 (10/11), pp. 1105-1122.

Fagerberg, Jan (2003), "Innovation: A Guide to the Literature", en The Oxford Handbook of Innovation, Oxford University Press, pp. 1-22.

Femia (2012), Programa Estratégico de la Industria Aeroespacial, Secretaría de Economía, México.

Freeman, Christopher (1987), Technology policy and economic performance: Lessons from Japan, Londres, Pinter Publishers.

Hernández, Eder (2016), Desafios de la formación de capital humano frente a modelos de capital emergentes, Instituto Politécnico Nacional (IPN).

Hernández, Jorge (2015), Empresas mexicanas en la cadena de valor de la IA-DCSIX Generación, México, Flacso.

Hernández, Juana (2010), Mecanismos de aprendizaje en la transferencia de conocimientos del modelo 400 y Global express. El caso de Bombardier Aeroespace, Querétaro, UAM-X.

Hualde, Arturo y Carrillo, Jorge (2007), La industria aeroespacial en Baja California: Características productivas y competencias laborales y profesionales, México, Colegio de la Frontera Norte.

Katz, Jorge (coord.) (1987), Technology Generation in Latin American Manufacturing Industries, The Macmillan Press Ltd., Londres. (2015), "Advanced manufacturing: where is America today?", Week, (10), pp. 26-30.

(2007), Cambios estructurales y ciclos de destrucción y creación de capacidades productivas y tecnológicas en América Latina, Chile, núm. 2007-6.

Killen, Catherine; Hunt, Roberta y Kleinschmidt, Elko (2008), "Learning investments and organizational capabilities: Case studies on the development of project portfolio management capabilities", International Journal of Managing Projects in Business, 1 (3), pp. 334-351. http://doi. org/10.1108/17538370810 883800

Lall, Sanjaya (1992), "Technological capabilities and industrialization", World Development, 20 (2), pp. 165-186. http://doi.org/10.1016/0305-750X(92) 90097-F 
López García, Santiago, et al. (2012), El clúster de la industria aeronáutica y espacial del país vasco: orígenes, evolución y trayectoria competitiva, España. Lundvall Bengt-Åke y Björn, Johnson (1994), "Sistemas nacionales de innovación y aprendizaje institucional", Comercio exterior, vol. 44, № 8, México, BANCOMEXT, pp 695-704.

Mill, John Stuart (1973), "A System of Logic, Ratiocinative and Inductive: Being a Connected View of the Principles of Evidence, and the M. of S. I. (8th ed. L. L. J. S. (1973)", A System of Logic, Ratiocinative and Inductive: Being a Connected View of the Principles of Evidence, and the Methods of Scientific Investigation, Londres, (8th ed. Lo).

Ngwenya-Scoburgh, Lizzie (2009), Organizational Learning: an exploration of the influence of capabilities and factors, Capella University.

Niosi, Jorge y Zhegu, Majlinda (2005), "Aerospace Clusters: Local or Global Knowledge Spillovers?", Industry \& Innovation, 12 (1), pp. 5-29.

Nonaka, Ikujiro y Takeuchi, Hirotaka (1999), La organización creadora de conocimiento: cómo las compañías japonesas crean la dinámica de la innovación, Oxford University Press.

Organización para la Cooperacion y el Desarrollo Económicos (OECD) (2006), Manual de Oslo, España.

Pavitt, Keith, Bell, Martin (1995), "The Development of Technological Capabilities", en T. W. Bank (ed.), Trade, Technology and International Competitiveness, Washington, pp. 69-101.

Ruiz, Ariel (2016), "La industria aeronáutica, la oportunidad de México", Entrevista con Calude Gobenceaux, Comercio Exterior, Bancomext, 6 (julio-septiembre).

Schumpeter, Joseph (1989), "Essays on Entrepreneurs, Innovations, Business Cycles and the Evolution of Capitalism", en N. J. edited by Richard V. Clemence, New Brunswick, pp. 253-231.

Secretaría de Economía (2012), Industria Aeronáutica en México, Dirección General de Industrias Pesadas y Alta Tecnología, México.

Secretaría de Hacienda y Crédito Público (SHCP) (2014), Empresario Pyme como usuarios de servicios financieros.

Soto, Enrique (2014), La construcción social del significado del trabajo. Un análisis comparativo en la industria aeroespacial de México y Canadá, El Colegio de la Frontera Norte.

Stacey, Ralph (2003), Strategic management and organizational dynamics: The challenge of complexity, Essex: Pearson Education Limited. 
Teece, David; Pisano, Gary y Shuen, Ami (1997), "Dynamic capabilities and strategic management", Strategic Management Journal, 18 (7), pp. 509-536.

Tomer, John (1987), Organizational capital: The path to higher productivity and well-being, Nueva York, Praeger Publishers.

Torres, Arturo (2006), "Aprendizaje y contrucción de capacidades tecnológicas", Journal of Technology Management \& Innovation, 1 (5), pp. 12-24.

Ulrich, David y Lake, Dale (1990), Organizational capability: Competing from the inside out, Nueva York, John Wiley \& Sons.

Urbina, Ernesto (2010), "Sectores emergentes y capacidades tecnológicas locales: acercamiento al caso de la industria aeroespacial en Sonora", Ideas Concyteg, 5 (64), pp. 1177-1186.

UTEPI (Unidad Técnica de Estudios para la Industria) (2007), Competitividad industrial del Ecuador, Quito-Ecuador, Ministerio de Industrias y Competitividad y Organización de las Nacionales Unidas para el Desarrollo Industrial (ONUDI).

Velásquez, Jorge y Yony, Ceballos (2008), "Estudio de un proceso de innovación utilizando la dinámica de sistemas", Red de Revistas Científicas de América Latina, El Caribe, España y Portugal Sistema de Información Cientifica, 21, pp. 127-159.

Villavicencio Daniel (coord.) (1994), Continuidades y discontinuidades de la capacitación, México, UAM-Xochimilco/Fundación Friedrich Ebert.

Villavicencio, Daniel; Rigas, Arvanitis y Minsberg, Liliana (1995), "Aprendizaje tecnológico en la industria química mexicana", Perfiles Latinoamericanos, Flacso, México, 4 (7), pp. 121-148.

Villavicencio, Daniel; Hernández, Juana y Souza, Leornardo (2013), "Capacidades y oportunidades para el desarrollo de la industria aeronáutica en Querétaro", en Flacso (ed.), La industria aeroespacial: complejidad productiva e institucional, México, pp. 49-91.

Zollo, Maurizio y Winter, Sindney (2002), "Deliberate learning and the evolution of dynamic capabilities", Organization Science, 13, pp. 339-351. 
